\title{
EL TRATAMIENTO DE LA TEMÁTICA HOMOSEXUAL EN CUATRO NOVELISTAS INGLESES: LAWRENCE, FORSTER, WAUGH Y STOREY (1914-1963)
}

\author{
Roberto A. Valdeón García \\ Departamento de Filología Anglogermánica y Francesa \\ Campus El Milán. Universidad de Oviedo
}

\begin{abstract}
Modernism imbued the English novelists of the first half of the 20th century with a sense of freedom in the themes and in the forms, putting an end to the stagnant narrative which had preceded them. However, social and religious repression affected these writers to the extent that certain themes were still prohibited and were only tackled with extreme care to maintain a delicate balance between their freedom and the norms of their society. In recent decades, intertextuality has attempted to analyse the literary contribution of some of these writers with reference to other texts and, in the most extreme cases, by avoiding any reference to their social background. In this article we aim to reconcile both views by offering a study of a controversial subject, male homosexuality, in the narrative of four English novelists of the 20th century (Lawrence, Forster, Waugh and Storey), which requires both an analysis of the humanistic relations among the texts and a direct reference to the society in which they were created.

Key words: English novel, 20th century, Homosexuality, Society, Forster, Waugh, Lawrence, Storey.

RESUMEN: La narrativa inglesa de la primera mitad del siglo XX nos ofrece, a través del modernismo, un nuevo espíritu de libertad basado en la renovación temática y estilística frente al anquilosamiento de los últimos exponentes del realismo. No obstante, la influencia de las instituciones sociales y religiosas aún se deja sentir en esta narrativa que aún ha de luchar por obtener un delicado equilibrio entre su concepto de libertad y las normas de la sociedad. En décadas recientes se ha intentado llevar a cabo una revisión intertextual de estos autores, que en los casos más extremos elimina toda referencia al contexto social en el que surgen. En este artículo pretendemos reconciliar el estudio de los aspectos sociales y una aproximación humanística al tema homosexual en las novelas de cuatro autores, Lawrence, Forster, Waugh y Storey.
\end{abstract}

Palabras clave: Narrativa inglesa, siglo XX, sociedad, homosexualidad, Lawrence, Forster, Waugh, Storey.

\section{Introducción}

La publicación en 1994 de la obra On Looking Into the Abyss de la norteamericana G. Himmelfarb, en el que se defiende la labor de críticos de la corriente humanísti- 
ca, tales como L. Trilling, quien dejó plasmado su pensamiento literario y filosófico en The Liberal Imagination ${ }^{1}$ frente a los partidarios de las teorías críticas del posmodernismo (Himmelfarb cita a Derrida y Foucault entre otros) dio lugar a una polémica en la que participó Mario Vargas Llosa. El crítico y novelista alaba en un artículo el trabajo de Trilling porque el crítico norteamericano estaba

convencido de que la historia humana era una empresa totalizadora, el progreso una realidad posible y la literatura una realidad de la imaginación con raíces en la historia y proyecciones en la moral, frente a quienes han relativizado las nociones de verdad y de valor hasta volverlas ficciones [en referencia a la crítica posmodernista], entronizando como axioma que todas las culturas equivalen y disocian la literatura de la realidad, confinando aquélla en un mundo autónomo de textos sin relacionarse jamás con la experiencia humana².

Aunque quizás las palabras de Vargas Llosa ofrezcan una visión demasiado drástica del análisis del discurso literario propuesto por los críticos posmodernistas, sí es cierto que determinadas propuestas de las últimas tres décadas han supuesto una entronización del texto y el intertexto, no tanto como lo introduce Kristeva (1967: 438-65), sino como lo asumen críticos posteriores como Worton \& Still (1990: 1-44), para quienes la obra literaria sólo puede entenderse a partir de la lecturas previas del autor, que aparecerían reflejadas en su obra, así como a través de un proceso de lectura por parte del lector, que como co-productor del texto, sólo podría entenderlo tomando en consideración su bagaje crítico-literario previo. En consecuencia señalan que "a delicate allusion to a work unknown to the reader, which therefore goes unnoticed, will have a dormant existence in that reading. On the other hand, the reader's experience of some practice or theory unknown to the author may lead to a fresh interpretation" (1990: 2). Igualmente narradores y/o críticos de la segunda mitad de siglo han disociado su obra del concepto de imitación y, en buena medida, de la realidad a la que cada autor pertenece de forma indisociable. El novelista y crítico inglés D. Lodge rechaza taxativamente la importancia que el componente imitativo pueda tener en su creación narrativa y prefiere hablar de narrativa en vez de novela, para eliminar la tradición de realismo que este término pueda suponer:

Realism as a literary form, or effect, of verisimilitude, is something we think of as very close to, if not quite synonymous with, the classical notion of mimesis or imitation, and we often describe the novel casually as a 'mimetic' literary form. In fact, of course, only drama is a strictly mimetic form (1990: 48).

\footnotetext{
1. Fue publicada por vez primera en 1940.

2. El artículo se publicó en el diario El País, el 27 de noviembre de 1994, y llevaba por título "Posmodernismo y frivolidad".
} 
Junto al rechazo de la tradición realista, también se ha dado un intento de destruir la idea del autor tradicional ligado a la realidad ${ }^{3}$, por lo que la novela quedaría reducida al texto y sus múltiples interconexiones con otros textos, es decir con el intertexto. Sin embargo, este somero y poco apropiado resumen de los aspectos más extremos de la crítica intertextual nos sirve de introducción al análisis de los textos que a continuación proponemos dentro de los parámetros que sugiere Kristeva (1968: 312-313) para obtener las coordenadas religiosas, sociales y educativas de las novelas de D. H. Lawrence (Lady Chatterley's Lover y Aaron's Rod), E. M. Forster (Maurice), E. Waugh (Brideshead Revisited) y D. Storey (Radcliffe) en un panorama que comprende desde el año 1914 (fecha en la que Forster acabó su novela) hasta 1963 (fecha en la que Storey publica Radcliffe) y que suponen los dos límites de la permisividad social y legal hacia la temática homosexual.

Si bien es cierto que el discurso de las novelas que hemos seleccionado podría analizarse únicamente en relación a textos no exclusivamente literarios, no puede obviarse el hecho de que los mismos forman parte de la tradición judeo-cristiana que afecta a los autores que estudiamos como miembros integrantes de una sociedad que se rige por códigos morales explicitados en leyes y formas sociales. Es precisamente en esta encrucijada donde el autor, no solamente el novelista, interviene con los instrumentos a su alcance para disociarse de la misma y transformarse, no en su representante, sino en su crítico ${ }^{4}$. No se trata, pues, de analizar la homosexualidad como fuente de inspiración literaria, como han hecho algunos críticos del posmodernismo (Barthes 1975; Foucault 1977b; Knight 1990), sino de examinar los textos de la época para dilucidar las posibles concomitancias existentes entre todos ellos, y alcanzar unas conclusiones sobre su relación con la sociedad en que surgieron.

Así pues, junto al marco crítico es necesario establecer el cuerpo de intertextos y su relación con su sociedad. En primer lugar hemos de tener en cuenta el valor represor de la sexualidad que la tradición judeo-cristiana ha ejercido sobre la sociedad inglesa y, por lo tanto, sobre la libre expresión artística de sus individuos, tanto a través de las Escrituras como del poder de las diferentes iglesias. D. H. Lawrence se expresó en este sentido en un ensayo escrito a finales de los años 1920, en el que señalaba una de las principales causas de represión en la dificultad que encuentran sus compatriotas para tratar el tema del sexo:

And the same is true of our sexual feelings; only worse. There, we start all wrong. Consciously, there is supposed to be no such thing as sex in the human

3. Ver, por ejemplo, la obra de M. Foucault Language, Counter-memory, Practice (1977: 113-38).

4. Consideración que sería rechazada por Lodge, quien asegura "I have always been concerned with the construction of a poetics of fiction, and the development of a literary history of the novel grounded in such a poetics rather than in content or contexts" (1990: 89). 
being. As far as possible, we never speak of it, never mention it, never, if we can help it, even think of it. It is disturbing. It is -somehow- wrong (...) When I was a lad of eighteen, I used to remember with shame and rage in the morning the sexual thoughts and desires I had had the night before (1950a: 99).

De esta forma cobran sentido las palabras de Trilling: "we are creatures of time, we are creatures of the historical sense" (1970: 191), ya que el autor forma parte de una sociedad que ejerce su influencia sobre él. Esa influencia, que para Foucault se asimila al advenimiento de la sociedad capitalista a partir del siglo XVII (1978:12), se ve reflejada en las leyes que penalizan determinadas conductas sexuales o consideradas obscenas. Por ello, cuando Forster señala que no ha escrito tanto como hubiera deseado 5 ha de comprenderse dentro del marco de la Inglaterra eduardiana, que prohibía la homosexualidad terminantemente y la castigaba con severas penas de prisión. El puritanismo victoriano había provocado ese rechazo de la homosexualidad masculina, tal y como se refleja en el polémico juicio que acabó con la carrera de Oscar Wilde en 1895, a la par que se negaba a reconocer la existencia del lesbianismo ${ }^{6}$. Esta situación de represión sexual se relaciona íntimamente con la existencia de un código estricto en cuanto a la publicación de léxico con connotaciones sexuales obscenas, el denominado Obscenity Act, que sólo a partir del juicio contra Lady Chatterley's Lover en 1960 sería gradualmente reformado. La prohibición de la homosexualidad con duras penas de cárcel, junto con la imposibilidad de utilización de vocabulario obsceno en la literatura de principios de siglo, muestra el claro talante hipócrita de la sociedad británica de la primera mitad de siglo, ya que como señala Aldous Huxley: "In silence, an act is an act is an act. Verbalized and discussed, it becomes an ethical problem, a casus belli..." (Huxley 1955: 103).

Así pues, los autores británicos se encontraban obligados, tanto por la moral cristiana de la sociedad a la que pertenecían como por sus leyes, a excluir la temática homosexual de su creatividad artística, independientemente del cariz que cada autor pudiese imprimirle. Por lo tanto, nos parece apropiado comenzar nuestro estudio con la denominada novela homosexual de Forster, ya que reúne las dos características que hemos señalado hasta este momento: la imposibilidad legal de publicar la obra (el propio autor la calificó de "unpublishable") y la represión sexual de que habló Lawrence, reflejada en el permiso de Forster de publicar su obra únicamente con carácter póstumo.

5. En una entrevista concedida a la BBC en 1959 con motivo de su octogésimo cumpleaños.

6. En este sentido ver la obra de G. Hugues Swearing: A Social History of Foul Language, Oaths and Profanity in English (1991: 228-233). Aunque la existencia de un menor número de términos despectivos para los homosexuales que para las lesbianas permite a Hugues confirmar una mayor tolerancia hacia las últimas, nuestra opinión es más bien la contraria: realmente refleja el total secretismo en que éstas se movían. 


\section{Instituciones represoras de la sociedad: educación y familia}

El tema homosexual en la narrativa de E. M. Forster ha sido, quizás, el más notorio en las dos últimas décadas debido a la publicación de su novela póstuma. Aunque como miembro del grupo de Bloomsbury (Bell 1974: 15), rechazara lo que denominaba supersticiones religiosas, en realidad se trataba de una toma de posición sin otras implicaciones personales7 . En esta tesitura el talento literario de Forster produce cinco novelas en las que se recrean relaciones heterosexuales, mientras que dedica, en secreto, un gran esfuerzo personal a la producción de la que se ha venido en llamar su novela homosexual ${ }^{8}$. Esta, como queda indicado, permanecerá oculta primero por razones legales y, más tarde, por razones personales, hasta su muerte. La aparición en 1971 de Maurice causará una pequeña conmoción en el mundo literario. La crítica en torno al autor, que había conocido su momento álgido en los años sesenta con las obras de Beer (1962), Crews (1962), Wilde (1964) y Stone (1966), para luego iniciar un proceso de decadencia en los años setenta, toma un nuevo impulso gracias a la polémica desatada por la temática de la novela: relataba la historia de un joven de clase media que descubre sus tendencias homosexuales.

La novela nos presenta a tres protagonistas masculinos: Clive Durham, Alec Scudder y Maurice Hall, aunque será el último quien dé título a la novela. Forster establece un triángulo de características muy peculiares: Maurice Hall entra en contacto con Clive Durham en Cambridge, donde ambos inician una relación afectiva de carácter homosexual. Más adelante, a través del segundo conocerá e iniciará una segunda relación afectiva con Alec Scudder, pues este último trabaja como guardabosques en la mansión de campo de Durham. En Maurice, Forster utiliza dos personajes dispares para mostrar al lector dos actitudes ante un mismo problema, y por ello caracteriza a Durhan como tipo mediante su apellido, frente al carácter precursor en Hall, la antesala a un cambio. Durham simbolizaría la tradición británica a través de sus universidades emblemáticas, en este orden, Oxford, Cambridge y Durham. Al mismo tiempo este nombre proporcionará el elemento irónico que le permitirá a Forster presentar a Clive como el más mediocre de los dos: Durham es la universidad en la que acaban aquellos que no han conseguido acceder a una de las otras dos.

7. Tal y como lo refleja, en el caso de Forster, la biografía escrita por P. N. Furbank E. M. Forster: A Life, en lo que se refiere a su represión sexual, sus ocasionales encuentros sexuales con miembros del mismo sexo y sus conflictivas relaciones sentimentales con otros personajes literarios o no de la época. Ver Furbank 1979: 220-255.

8. Expresión que rechazo en mi tesis doctoral, publicada bajo el título de E. M. Forster: una revisión intertextual. En ella defiendo la importancia de considerar esta novela como integrante de un sexteto de obras que expresan el mismo sentir humanista de su autor por el hombre en todas sus dimensiones: "No obstante, en Maurice descubrimos, al margen de su tema homosexual, todas las cualidades de la narrativa de Forster, así como la mayor parte de sus intereses reflejados fielmente" (Valdeón 1995: 35). 
La influencia que las instituciones educativas ejercerán sobre el protagonista parte de los primeros capítulos, en los que el protagonista es aún un niño y se encuentra en la escuela. Al pertenecer a una familia de clase media-alta y por su condición de alumno mediocre, Maurice no tiene opción a una de las escuelas privadas tradicionales (Eton o Harrow), pero sí al menos de acudir a una escuela privada, a una escuela respetable, que le servirá de paso previo a su traslado posterior a Oxbridge. Elimina Forster todo comentario con respecto a la primera institución educativa a la que asiste el protagonista; ésta aparece definida en torno al personaje del director, para el que el autor escoge un nombre de resonancias bíblicas, Mr Abrahams. Forster añade además la presencia de Mrs. Abrahams con el fin de crear estabilidad:

Lest discipline should suffer, it took place just before the holidays, when leniency does no harm, and indeed it seemed more like a treat at home than school, for Mrs Abrahams, the Principal's wife, would meet them at the tea place with some lady friends, and be hospitable and motherly.(Forster 1972: 15).

La escena es significativa en tanto en cuanto recrea la estructura social dominante de la época, y así los valores tradicionales son encarnados por el director y su esposa. Con el nombre que Forster ha escogido para el director sitúa a ambos en la esfera del poder ancestral de la moral religiosa cristiana encarnada en dos de sus descendientes modernos: Abraham, que fue el patriarca del pueblo de Israel, es un nombre que quiere decir "padre de multitudes"9. De esta forma, el Mr Abrahams de Forster se sitúa a la cabeza de la escuela, cuidando la salud, educación y moral de sus alumnos. A su lado, Mrs. Abrahams aparece caracterizada como la nueva tierra prometida ("hospitable") y madre ("and motherly"). Forster reitera la conexión de la pareja con el pasado, al definir al director como "a preparatory schoolmaster of the old-fashioned sort", puesto que debe transmitir la noción de un mundo de orden a sus alumnos, orden que nos viene dado por la amplia gama de adjetivos y sustantivos que hacen referencia a ello: "discipline", "leniency", "misbehave".

En este mundo estratificado en base a la tradición cristiana comienza su andadura el protagonista de Maurice, en el que otros personajes secundarios inician a Maurice en su distanciamiento de las estrictas costumbres eduardianas, aunque de una forma torpe e incomprensible. De ellos Mr Ducie es el primero en hacer su aparición a través de una pretendida lección sobre la vida y el sexo: "What did Mr Abrahams say? Told you you were a miserable sinner, I hope" (p.17). Mr Ducie establece de esta forma tres

9. La referencia a textos bíblicos es, pues, irrefutable. Ver, por ejemplo, Génesis 12 y 15 del Antiguo Testamento y Romanos 4 del Nuevo. Este último es especialmente significativo como podemos comprobar en la versión de la Biblia conocida para Forster: "What shall we say then that Abraham our father, as pertaining to the flesh, hath found? For if Abraham were justified by works, he hath whereof to glory; but not before God. For what saith the scripture? Abraham believed God, and it was counted unto him for righteousness." 
tipos de relaciones: una actitud irónica con respecto al director, un distanciamiento con respecto a sus métodos y una aproximación a su joven interlocutor. Así surge una complicidad que permitirá la comunicación entre los dos personajes. La respuesta del joven Maurice no se hace esperar y, resulta, como cabría esperar, positiva: "The boy laughed. He did not understand Mr Ducie, but knew that he was meaning to be funny" (p. 17).

En la segunda etapa del Maurice niño, Forster nos traslada al hogar materno y nos retrata una escena familiar de estabilidad y ausencia de lucha. La relación existente entre madre e hijo queda marcada por los dos nombres con los que el uno se dirige al otro respectivamente: "Morrie" y "Mummie". A pesar de la aparente tranquilidad, el autor anticipa la problemática de su protagonista a través de un procedimiento que ya había sido señalado por el crítico P. Burra10, y más tarde estudiado en nuestro trabajo sobre la narrativa del autor inglés, la inclusión de insinuaciones de un conflicto futuro que sólo se advierten en una segunda lectura: Maurice pregunta con insistencia por el paradero de George, el joven jardinero que había trabajado en la casa. Su inquisitoria insistencia en conocer el paradero de George muestra una inquietud que ni su madre ni sus hermanas son capaces de tranquilizar: "Where's George?", "Mother, where's George?" y "Why did George leave?" (pp. 21-22). En este primer momento de aproximación a Maurice, niño, el autor lo hace desde una perspectiva objetiva: el narrador se limita a repetir preguntas y respuestas y a observar reacciones. Así, frente a la estabilidad del colegio, los primeros indicios de ruptura nos llegan cuando finalmente Maurice comienza a llorar: "The little boy was in tears" (p. 22). Al final del capítulo comenzamos a vislumbrar una personalidad compleja a través de las palabras del narrador:

Something stirred in the unfathomable depths of his heart. He whispered, "George, George." Who was George? Nobody, just a common servant. Mother and Ada and Kitty were far more important. But he was too little to argue thus. He did not even know that when he yielded to this sorrow he overcame the spectral and fell asleep (p. 24).

El narrador establece la situación en la que va a producirse la paulatina transformación del personaje mediante un párrafo que se contrapone a las descripciones que

10. Burra señala: "Mr Forster has developed the art of clues and chains to an unusual extent. In its simplest form it consists of throwing in hints that are a preparation for events to follow probably much later. They are generally so casually introduced that we hardly observe them; hence a full appreciation of his novels depends absolutely on a second reading. For example, the deaths of Mrs Wilcox, Leonard Bast and Mrs Moore appear when we come upon them to be too sudden for credibility (...). In point of fact, they are quite deliberately prepared for by earlier remarks ..." en "The Novels of E. M. Forster", Nineteenth Century and After, CXVI, Noviembre 1934; recopilado en E. M. Forster: A Passage to India, editado por Malcolm Bradbury, Casebook Series, MacMillan, Londres, 1970. Luego tratado en Valdeón 1995: 73-85. 
hemos visto hasta este momento, a excepción de la descripción de la casa de Mrs Hall, donde nos encontramos con tres expresiones positivas: "mother, comfortable villa, pines". Familia, hogar y naturaleza. En este párrafo, con el que concluye el capítulo 2, el narrador utiliza un gran número de términos negativos para definir el alterado estado de ánimo del protagonista: "unfathomable depths, sorrow, the spectral", términos que nos remiten a la novela gótica inglesa, y que resultan efectivos para contrastar el escenario a los sentimientos del joven.

Similar es el tratamiento inicial que David Storey hace de su protagonista en Radcliffe, la historia de un joven que ha de enfrentarse, al igual que Maurice, a una relación con otro joven, consciente de las dificultades sociales que ello pueda suponer. Las concomitancias con la novela de Forster se inician en el primer capítulo, al situar a Radcliffe en una institución educativa, en este caso una escuela pública (aunque se señala su paso por otra privada). Al igual que Maurice, el joven Radcliffe queda caracterizado como un niño normal, a pesar de lo cual llama la atención del resto de los alumnos:

The Headmaster brought the new boy into the classroom several weeks after the term had begun. He stood alone, the centre of the children's curiosity, as the class-teacher, a squat, kindly-faced matron, talked quietly with the Headmaster by the open door. At first he returned the solemn gaze of the children, but as time passed he began to blush and look urgently away (...). Eventually several of the children burst out laughing at his plaintive isolation (Storey 1965: 7).

Aunque la posición social de su protagonista es similar a la de Maurice (representa a una aristocracia decadente), la escena difiere considerablemente de la novela de Forster: "The war confirmed the industrial domination of this land and in 1921 the last of the farmland was sold" (p. 14). Storey no retrata un personaje atormentado en su infancia por deseos sexuales claramente identificados, sino que el Radcliffe niño recién llegado inicia una relación de camaradería alejada del bucolismo de las escuelas privadas inglesas, de las universidades de Oxbridge o de las mansiones de las clases medias-altas. La relación, como se ha señalado "goes beyond the social issue of class" (Stevenson 1986: 132), y surge en un medio social hostil, definido en torno a la desaparición de la Inglaterra ideal, que ha cedido terreno al progreso industrial:

During the following decade the red houses of the estate crept slowly up the broad escarpment, absorbing first the stone cottages of a heathland village, then several towering oak trees and a wide avenue of elms, and finally surrounding the Place and its attendant church within a denuded perimeter of shrubbery and trees (p. 14).

No obstante, y a pesar de que el autor defina las relaciones entre Radcliffe y su compañero de clase Tolson con palabras sin connotaciones de ningún tipo, tales como 
"classmates", sí que anticipa la atracción que el protagonista llegará a sentir en un futuro por Tolson: "He began to pay Tolson a solemn yet distant attention which, through a kind of surly aloofness, was obscurely returned" (p. 12). Es decir, la aproximación entre los dos personajes se caracteriza a través de una palabras de connotaciones negativas, "obscurely", paralelas a las que habían definido la ansiedad de Maurice Hall ante la partida del joven jardinero. La utilización de dicha palabra tomará significado paulatinamente a medida que la evolución de su relación se consolida: "their relationship became the vehicle of his first excursion into that new and intimidating world outside" (p. 35), "Tolson had no father" (p. 36), "without the compensation and protection of Tolson he found himself even more isolated than in his first year at the elementary school" (p. 43).

\section{La represión de la sexualidad: la camaradería como alternativa}

No obstante, las concomitancias entre el protagonista de Forster y el de Storey pronto comienzas a diluirse. Para la definitiva caracterización de su personaje Forster incluye dos sueños, y aunque no existe constancia de que el autor conociera La interpretación de los sueños, ambos responden a las coordenadas que Freud define:

Por su carácter de realización de deseos se dividen los sueños en tres clases: en primer lugar, aquellos que muestran francamente un deseo no reprimido. En segundo lugar, los que exteriorizan disfrazadamente un deseo reprimido; esto es la mayoría de aquellos que necesitan del análisis. Y en tercer lugar, aquellos otros que, si bien representan un deseo reprimido, lo hacen sin disfraz alguno o con un disfraz insuficiente. Estos últimos sueños suelen acompañarse de angustia ...(Freud 1988: 108).

Los dos sueños en Maurice hacen referencia a una única preocupación, y ambos han de reflejar el subconsciente del personaje. En el primero, aparece George, personaje que, aunque presente en la mente del protagonista, siempre permanece ausente en la obra: el joven jardinero corre hacia él, desnudo, siendo la imagen del deseo aún por descubrir. Por otro lado, el segundo sueño resulta más difícil de definir: el narrador omnisciente nos describe un sueño confuso, que subraya la confusión del propio protagonista. En el breve relato, tres elementos se confunden en una gradación muy significativa: la tradición cristiana, la herencia clásica y el racionalismo (Valdeón 1995: 44-57).

George headed down the field towards him, naked and jumping over the woodstacks. 'I shall go mad if he turns wrong now,' said Maurice, and just as they collared this happened, and a brutal disappointment woke him up.

He scarcely saw a face, scarcely heard a voice say 'That is your friend,' and then it was over, having filled him with beauty and taught him tendernes. He 
could die for such a friend, he would allow such a friend to die for him (...). Soon afterwards he was confirmed and tried to persuade himself that the friend must be Christ. But Christ has a mangy beard. Was he a Greek god, such as illustrates the classical dictionary? More probable, but most probably he was just a man (pp. 25-26).

A pesar de lo comedido del retrato de Maurice como homosexual, el hecho de que George aparezca desnudo en el sueño no deja lugar a dudas, y apoya, desde los primeros capítulos, la sospecha que el propio Forster albergaba con respecto a su novela y a su época: su tema era demasiado escabroso para publicarse. Es más, los dos sueños que hemos reproducido parcialmente se conforman perfectamente al tercer tipo de Freud: Forster otorga a su personaje adolescente rasgos de infelicidad por segunda vez, tras la decepción por la partida de George, por lo que aparece la angustia de la que habla Freud: "Misery was somehow mixed up with all this happiness" (p. 26). La ignorancia de lo físico se ha apoderado de nuestro personaje, ahogado por un sentimiento religioso de culpabilidad: "He supposed some special curse had descended on him" (p. 26). La ciencia y la literatura ofrecen claras referencias a una preocupación vital subyacente en la época que adquiría dos formas diferentes, ambas susceptibles de rechazo: la obra de Freud sufrió el rechazo de sus coetáneos (los seiscientos volúmenes de la obra no llegaron a venderse) y Forster censuró su propia creación.

La represión sexual se agudiza con la intervención precisamente del estamento científico, personificado por el Dr Barry, un amigo de la familia. Una pregunta suya va a profundizar la crisis de Maurice, puesto que introduce un nuevo elemento de distorsión: "And after you're let in by your father's old partner, what? A pretty wife?" (p. 29), que enfrenta a Maurice no con su conflicto personal, sino con las expectativas, la moral y la tradición de la sociedad en la que nació y a la que desea pertenecer, por lo que los comentarios subsiguientes del amigo de la familia profundizan el conflicto de Maurice: "Dr Barry went on lecturing him, and under the cover of a friendly manner said much that gave him pain" (p. 30), palabras que agudizarán su sentimiento de no-pertenencia: "Maurice became modest and conscious of sin: in all creation there could not be one as vile as himself" (pp. 31-32).

Estas palabras de Forster muestran un claro paralelismo entre este autor y D. H. Lawrence, quien, también a principios de siglo, escribía sobre los británicos y el sexo, y recogía sus propios sentimientos de culpabilidad cuando, como adolescente, recordaba sus deseos sexuales de la noche anterior: "And it was a long time before I was able to say to myself: I am not going to be ashamed of my sexual thoughts and desires, they are me myself, they are part of my life" (Lawrence 1950a: 100). Estos mismos sentimientos afectan al personaje de Forster, que aparece envuelto por los primeros síntomas de un complejo de culpabilidad con respecto a unos sentimientos que considera desviados de la moral tradicional, y que le han proporcionado las instituciones educativas, religiosas y familiares que la sociedad pone en marcha para todos sus nuevos miembros. Sin embargo, Forster, al igual que había hecho Lawrence en su ensayo 
autobiográfico, escribe una de las líneas más efectivas de la novela al caracterizar a su protagonista como inmerso en un proceso de auto-reflexión aún sin implicaciones finales directas: "he saw that while deceiving others, he had been deceived" (p. 32). Este punto de inflexión en la novela de Forster va a suponer un primer momento de revelación y de liberación al desplazar sus sentimientos de culpabilidad a una sociedad que impone normas de intolerancia hacia quienes se desvían de conductas consideradas moralmente aceptables.

Esta focalización en el conflicto personal de Maurice no tiene una total equivalencia en la otra novela de temática abiertamente homosexual, Radcliffe, puesto que el protagonista establece inmediatamente una relación física con Tolson, y aunque el autor prefiere concluir el capítulo 6 antes de mostrarse más explícito, no cabe duda de que las relaciones de los dos protagonistas toman un marcado carácter físico en los dos últimos párrafos:

'If we take everything off we'll be as warm as anything under the blankets. Shall we do that?' He began to take off his thin, mud-stained pyjamas, watching Leonard closely, almost threatingly. Slowly Leonard began to remove his jackets, still gazing down at the ground.

A moment later, naked, Tolson came and touched him. 'Hold me. Hold me! he said, his lips buried in the hair behind Leonard's ear (Storey 1965: 67).

No obstante sí que existen coincidencias en el rechazo a las conductas aceptadas socialmente en ambos personajes, aunque en el caso del personaje de Storey, este rechazo se presente de forma tajante y, finalmente, brutal. En esta escena, Radcliffe aún duda de la situación a la que Tolson le conduce. La utilización de "obscurely" se complementa con una descripción distanciada de la actitud de Tolson hacia Radcliffe: "threatingly" y "closely", que definen el carácter iniciático de la relación del primero con el segundo (igualmente similar al iniciador Clive Durham). Es, pues, un mundo masculino, alejado del mundo femenino del hogar. En la dicotomía masculino/femenino de Radcliffe, éste no aparece privado de padre, aunque sí se trata de un personaje cuyas debilidades e indecisiones afectan directamente al protagonista: "At the age of thirty John Radcliffe had decided that his distaste for the society in which he lived was so complete that he could no longer reconcile himself to being a member of it" (p. 18). Esta ruptura, asociada al rechazo de anteriores sentimientos de temor religioso ("a superstitious fear of God") o al abandono de su posición dentro de la sociedad, anticipa la imposibilidad de Leonard Radcliffe de amoldarse a la sociedad que su padre ya odiaba de joven.

Junto a la negación de sociedad, educación y religión, se produce un rechazo de lo femenino como ajeno a la relación hombre-hombre. En Radcliffe éste tiene lugar en escenas tan significativas como la previa al primer encuentro de carácter claramente físico entre los protagonistas, en la que hombres y mujeres están montando unas tiendas y Tolson pide al capataz la expulsión de la mujer que está molestando a su amigo 
Radcliffe: "Can you ask this girl to go, sir? (...) She won't leave us alone" (p. 72). Las palabras de Tolson resultan aún más significativas dentro de su contexto: Radcliffe, después de años sin ver a Tolson, acaba de conocer a la mujer y a los hijos de éste, pero el narrador los presenta como figuras marginales, intrascendentes para la tragedia que comienza a desarrollarse.

En este aparente ideal de camaradería masculina y rechazo de lo femenino, Storey recibe la influencia y recrea situaciones que Lawrence había imaginado y descrito décadas atrás. En Aaron's Rod, Lawrence crea un protagonista masculino que busca su identidad y su individualidad como ser humano, así como en su relación con el arte. La situación no puede ser más alejada de la trama de Storey. En el capítulo 9, la estrecha amistad de Aaron Sisson con Rawdon Lilly lleva al último a cuidar de un Aaron enfermo de gripe. La entrega de Lilly hacia Aaron es tal que Lawrence la describe así:

Quickly he uncovered the blond lower body of his patient, and began to rub the abdomen with oil, using a slow, rhythmic, circulating motion, a sort of massage. For a long time he rubbed finely and steadily, then went over the whole of the lower body, mindless, as if in a sort of incantation. He rubbed every speck of the man's lower body - the abdomen, the buttocks, the thighs and knees... (Lawrence 1950b: 118).

En este breve párrafo, sin apenas consecuencias para la relación entre los dos protagonistas, el narrador nos ofrece el más claro ejemplo de su narrativa de una relación masculina de claras referencias homosexuales. Ésta se explicita a través de la entrega de Lilly hacia Aaron, y, aunque la pretensión del autor parece tendente a retratar una escena de amistad hasta sus últimas consecuencias a través de palabras que expresan ausencia de sentimientos ("using a slow, rhythmic, circulating motion", "finely and steadily", "mindless"), éstas se confunden con otros términos mucho más explícitos con claras connotaciones sexuales tales como "incantation", "rubbed", la utilización de la expresión "lower body" en tres ocasiones y la descripción de la misma con un detalle que muestra el claro interés del personaje por la apariencia física de Aaron, "the blond lower part".

La referencia a los genitales masculinos es un recurso que Lawrence utiliza con frecuencia para acentuar el contenido sexual de la situación, incluso si, en ocasiones, no resulta tan obvio. En su obra polémica por excelencia, Lady Chatterley's Lover, Lawrence es precursor e iniciador de una narrativa libre de prejuicios sexuales, sociales y lingüísticos sin parangón en la historia de la literatura inglesa. El propio narrador realiza una declaración de principios en dicha novela:

The novel, like gossip, can also excite spurious sympathies and recoils, mechanical and deadening to the psyche. The novel can glorify the most corrupt feelings, so long as they are conventionally 'pure'. Then the novel, like gossip, 
becomes at last vicious, and, like gossip, all the more vicious because it is always ostensibly on the side of the angels (Lawrence 1960: 105).

En este claro ataque contra la tradición de la narrativa inglesa, Lawrence rechaza esas restricciones que impiden la libre expresión del autor, que provocan la prohibición en el uso de determinado grupo de palabras del léxico inglés. Lawrence pone fin a todas estas restricciones a través de la relación de una mujer noble con el hombre casado de clase baja, contraviniendo las normas morales y sociales de la época, al mismo tiempo que pone fin a las convenciones lingüísticas que impedían el uso de palabras consideradas tabú. Junto a esto, la caracterización del protagonista, Mellors, nos muestra a un hombre débil, especialmente apegado a su superior en el ejército ("the colonel who had loved him and whom he had loved", p. 147), su relación de inferioridad con respecto a su mujer ("The prig and the bully!", p. 207) y excesivamente receloso de las mujeres ("Seems to me they're nearly all Lesbian", p. 212). Sólo la relación fortuita e inesperada con Lady Chatterley le pone en situación de relativa superioridad frente a una joven aristócrata e inexperta, incapaz de encontrar sentido a su monótona existencia. Precisamente esa relación física se traduce en explícitas descripciones de lo masculino, no desde la perspectiva masculina, sino de lo masculino desde la perspectiva femenina, cuya intensidad se aprecia con todo detalle:

He slipped out of bed with his back to her, naked and white and thin, and went to the window, stooping a little, drawing the curtains and looking out for a moment. The back was white and fine, the small buttocks beautiful with an exquisite, delicate manliness, the back of the neck ruddy and delicate and yet strong.

(...)

He was ashamed to turn to her because of his aroused nakedness.

He caught his shirt off the floor, and held it to him, coming to her.

(...)

He dropped the shirt and stood looking towards her. The sun through the low window sent a beam that lit up his thighs and slim belly and the erect phallos rising darkish and hot-looking from the little cloud of vivid gold-red hair (p. 218).

Estos párrafos y las páginas siguientes nos muestran al Lawrence del hombre apocalíptico del que habla Kermode ${ }^{11}$. Se trata de un protagonista masculino entregado al sexo como única forma de redención en un mundo cruento, que intenta forjar su individualidad en torno a su propio cuerpo. La deificación del cuerpo se manifiesta, pues, en esa entrega al otro ser humano, que glorifica la visión de lo físico y le libera

11. Kermode hace referencia a la visión apocalíptica de Lawrence en un mundo que consideraba en crisis a causa del progreso y la revolución industrial. El hombre abocado al Apocalipsis sólo podría redimirse a través del sexo, tal y como, según este crítico, aparece reflejado en esta novela, pero también en Women in Love y The Rainbow (Kermode 1968). 
de la negación de lo sexual. Es, pues, sintomático de Lawrence la creación de un mito físico donde prima la visión del cuerpo masculino, las referencias a los genitales masculinos en situaciones de liberación, tanto si se trata de un relación heterosexual, como en Lady Chatterley's Lover, o de amistad masculina exclusivista, como en Aaron's Rod, donde además el propio título conlleva una referencia sexual encubierta ${ }^{12}$.

\section{La confusión de género como alternativa}

Frente a la sexualidad de los personajes masculinos de Lawrence, de clase media-baja o baja, desprovista gradualmente de tabús y acentuada por la búsqueda de la verdad a través del cuerpo, se opone diametralmente la caracterización del personaje de Risley en Maurice, un estudiante de pose afeminada que desafía la tradición de Cambridge con unas ideas singulares acerca del arte, la sociedad y la vida. Aunque Maurice no llega a comprender a Risley, siente una atracción hacia el personaje difícil de definir. A Maurice le desagrada el amaneramiento de Risley, una característica que le convierte en un estudiante temido y despreciado por sus compañeros. Risley es un personaje-tipo que se asemeja sobremanera a Anthony Blanche de la novela de Evelyn Waugh Brideshead Revisited. Varios son los puntos en común entre los dos personajes, al margen de su homosexualidad: ambos tienen ascendencia francesa, ambos se mueven en círculos bohemios, ambos participan de la nueva moralidad imperante y, sobre todo, ambos suponen un nexo de unión entre los protagonistas de las dos novelas, aunque Waugh, más inquieto por la crítica hacia los movimientos artísticos del momento, logra una caracterización compleja y completa de Anthony Blanche en torno a sus creencias, como representante del esteticismo de principios de siglo. En este sentido, Green señala: "Anthony also flouts middle-class seriousness and conscientiousness; he also lives for beauty and wit; he also inhabits exotic terrains of the imagination" (1984: 124-125). En Maurice, sin embargo, Risley sirve de resorte para poner en contacto a su protagonista con Clive Durham.

El narrador nos lo presenta a través de una descripción física que no otorga a este nuevo personaje ninguna cualidad especialmente atrayente: "he was a small man very small - with simple manners and a fair face” (p. 37) para que la atracción de Maurice hacia Clive Durham surja por motivos no meramente físicos:

He [Fetherstonhaugh] and Durham were in for the same Tripos, and talked shop while Maurice listened. His excitement had never ceased. He saw that Durham was not only clever, but had a tranquil and orderly brain. He knew what he wanted to read, where he was weak and how far the officials could help him. He

12. En inglés Aaron's Rod es un antiguo eufemismo para el órgano sexual masculino. Ver, por ejemplo, Goldenson, R. \& K. Anderson 1994. 
had neither the blind faith in lectures and tutors that was held by Maurice... (Forster 1972: 39).

Se hacen evidentes las características intelectuales de Clive que han de atraer a Maurice en el futuro, así como la posición de admiración y, hasta cierto punto, sumisión que va a adoptar en los primeros estadios de su amistad con Durham. El narrador añade otras consideraciones que Maurice sería incapaz de intuir, pero que ayudan al lector a situar al personaje. En este momento inicial el interés de Maurice no se centra en la belleza de un compañero de otro año y otro colegio, sino en su personalidad. El paralelismo que habíamos establecido entre Maurice y Brideshead Revisited vuelve a surgir en la escena que sirve de punto de contacto entre los protagonistas, Charles Ryder y Sebastian Flyte. Ryder, en primera persona, recuerda su primer contacto visual con el también aristócrata, al igual que Durham, Sebastian Flyte: "I knew Sebastian only by sight long before I met him. That was unavoidable for, from his first week, he was the most conspicuous man of his year by reason of his beauty, which was arresting, and his eccentricities of behaviour, which seemed to know no bounds" (Waugh 1952: 28).

Sin embargo, y a pesar de las similitudes entre los dos encuentros, el carácter de los dos párrafos difiere notablemente tanto en su forma como en su finalidad. Mientras que Forster se nos presenta como narrador omnisciente y aparentemente objetivo, Waugh crea un narrador en primera persona que recuerda el pasado con nostalgia. Éste procura justificar su propia y subjetiva fascinación, mientras que el narrador de Maurice tiene como finalidad destacar objetivamente unas cualidades; así, mientras Sebastian Flyte es definido como "the most conspicuous man of his year", Clive Durham se destaca por su mediocridad física, tanto en su estatura como en su aspecto. Forster prefiere que su protagonista se interese por Clive Durham en tres estadios (Valdeón 1995: 52) hasta llegar al reencuentro, tímidamente físico, con Clive, quien le confiesa su amor. En el primero de esos estadios Maurice, y tras una discusión con su madre por motivos religiosos, renuncia, finalmente, a la frágil fe que había profesado hasta el momento:

He realized that he had no sense of Christ's existence or of His goodness, and should be positively sorry if there was such a person. His dislike of Christianity grew and became profound (...). They were both puzzled, and Maurice, although he had lost and yielded all his opinions, had a queer feeling that he was really winning (Forster 1972: 49).

El siguiente estadio, una vez liberado de la presión religiosa, también tiene lugar en la casa materna. Se trata de una prueba física en la que Maurice coge de la mano a una joven en un intento de representar el papel de "domineering male". El encuentro con la joven Miss Olcott y la necesidad de mostrar sus cualidades varoniles sufren un serio contrapunto ante la reacción del objeto de su atención: "His touch had revolted 
her. It was a corpse's" (Forster 1972: 53). Maurice no dará mayor importancia al incidente, pero la reacción de Miss Olcott así como sus palabras, en su desproporción, insinúan por vez primera y de forma clara la imposibilidad de Maurice para establecer una relación heterosexual, aunque el protagonista, a primera vista, no parezca verse mayormente afectado por el brusco rechazo de la joven.

Sin embargo, es muy significativo el hecho de que los dos incidentes que nos llevan al clímax en Cambridge estén relacionados con dos encuentros con personajes femeninos, la madre de Maurice como pasado y una mujer que simboliza el futuro, y que ambos incidentes se resuelvan de forma negativa: rechazo a la tradición familiar y religiosa, rechazo a la posible vida heterosexual dentro de esa tradición. El papel de la mujer como influencia negativa es fundamental en la caracterización del protagonista. Esta influencia no es privativa de Forster, ya que todos los ejemplos de narrativa inglesa que hemos mencionado nos ofrecen a personajes masculinos en conflicto con personajes femeninos. Hemos mencionado el caso del rechazo de Radcliffe y Tolson de una joven en el capítulo 7 de Radcliffe, pero la presencia amenazadora de las mujeres continúa en toda la novela a través de la mujer de Tolson, que supone una barrera entre ellos, o de la hermana de Radcliffe en una violenta escena física entre ambos en el capítulo 20: "It possessed, isolated like this, a strange tough remote sense of threat. It was his sister, Elizabeth" (p. 210).

Lawrence también nos ofrece frecuentes muestras de conflictos entre personajes masculinos y femeninos en sus novelas. Tanto en Aaron's Rod como en Lady Chatterley's Lover, los protagonistas masculinos han fracasado en sus experiencias matrimoniales y reconocen su debilidad de carácter con respecto a las esposas. Estas sólo cumplen una función caracterizadora ${ }^{13}$ y sólo contamos con las referencias del narrador o del propio personaje. La indefensión de Mellors frente a las mujeres y su misoginia es evidente en el comentario mencionado con anterioridad. La influencia negativa de personajes femeninos sobre la personalidad de los personajes masculinos es también innegable en Sons and Lovers, donde el padre de la familia Morel carece de importancia frente a la prepotencia de una madre, que obstaculiza con su carácter posesivo las relaciones de sus hijos con otras mujeres, por lo que acaban fracasando. Estas relaciones viciadas madre-hijos son obvias en numerosas ocasiones: "The mother and son walked down Station Street, feeling the excitement of lovers having an adventure together" (Lawrence 1948: 117)14 y, al igual que Forster, los sueños también ocupan un lugar señalado en el desarrollo de los personajes, en esta ocasión para inter-

13. Y, por lo tanto, cualquier juicio moral sobre el hecho de que abandone a su esposa es irrelevante, puesto que se trata, como señala F. R. Leavis, de un "tipo familiar de punto muerto que frustra la vida" (1974: 47).

14. La funcionalidad caracterizadora de la mujer aparece en sus otras novelas. En The Rainbow, Tom Brangwen se nos presenta en las primeras páginas de la siguiente forma: "He was drunk at a public house, he went upstairs with a prostitute who seduced him. He was then nineteen" (1949: 19). 
pretar el papel opresor y represor de la madre15.

Todos estos puntos son, pues, coincidentes en las novelas que hemos mencionado: el papel represor de la religión, la presión de la sociedad a través de la educación y el papel negativo de las mujeres que transmiten los parámetros encorsetados de la sociedad. Sin embargo, tan sólo Maurice focaliza el conflicto interior del protagonista a partir de las dudas sobre su orientación sexual. El narrador ofrece el juego físico entre ambos de la siguiente manera:

His head fell back, and he forgot the pressure of Durham on his knee, forgot that Durham was watching his turbid agony. He stared at the ceiling with wrinkled mouth and eyes, understanding nothing except that man has been created to feel pain and loneliness without help from heaven.

Durham streched up to him, stroked his hair. They clasped one another. They were lying breast against breast soon, head was on shoulder, but just as their cheeks met someone called 'Hall' (...) Both started violently (p. 55).

Y aunque la escena coincide con una situación de Brideshead Revisited, en la que Evelyn Waugh nos presenta una excursión campestre de los dos protagonistas: "[we] lay on our backs, Sebastian's eyes on the leaves above him, mine on his profile (...) That day was the beginning of my friendship with Sebastian, and thus it came about, that morning in June, that I was lying beside him in the shade of the high elms watching the smoke from his lips drift up into the branches" (pp. 28 y 34) las implicaciones para el protagonista adquieren un carácter diametralmente opuesto, ya que esta situación nos conduce a otra escena en la que ambos parecen negarse a aceptar el inocente juego de caricias como una declaración de sentimientos. Sin embargo, ésta concluirá de forma magistral con la confesión inesperada e impaciente de Clive, tanto para el protagonista como para el lector: "I love you", a la que sigue la reacción escandalizada del propio Maurice:

Maurice was scandalized, horrified. He was shocked to the bottom of his suburban soul, and exclaimed 'Oh, rot!' The words, the manner, were out of him before he could recall them. 'Durham, you're an Englishman. I'm another. Don't talk nonsense (...) it's the only subject absolutely beyond the limit (p. 56).

El narrador otorga un tono dramático que se contrapone a la mogijata reacción de Maurice al expresar su horror inicial ante la confesión de Clive. Este horror parte de dos premisas: su educación y el esnobismo de clase. La educación que hemos comentado con anterioridad, queda reflejada en su mentalidad de clase media y el

15. Ver el análisis de Leavis en la página 64 y siguientes. La figura de la madre como opresora aparece magníficamente caracterizada en la novela de Christopher Isherwood All the Conspirators, publicada en 1928. Es especialmente significativo el capítulo 11. 
narrador no duda en denostar el origen de la conmoción: "his suburban soul". En un segundo plano están las palabras del propio Maurice, quien ante el cariz que toma la situación hace uso de su último resorte: el esnobismo de clase. Para ello recuerda a Clive que ambos son ingleses y que, en consecuencia, acaba de mencionar un tema intocable. Lo significativo de la intervención de Maurice radica en el hecho de que, en un primer momento, sus tambaleantes principios se erigen triunfantes, pero Forster ha sido cuidadoso: es un triunfo temporal basado en una reacción, paradójicamente, pasional, en la que prima la represión de sentimientos. El autor escoge las palabras y los pretextos que Maurice va a pronunciar de manera cuidadosa, puesto que todos ellos resultan de escaso fundamento racional: "we're Englishmen (...) it's the only subject absolutely beyond the limit as you know (...) the worst crime in the calendar (...) a rotten notion really." Estos argumentos, aunque comprensibles dentro de la sociedad británica de principios de siglo, parten del rechazo irracional, del reconocimiento de una realidad social aceptada como verdad total ("as you know") y de la propia educación de clase.

Junto a la situación creada entre Maurice y Clive, Forster nos ofrece la evolución de Maurice a través de tres estadios: un momento de pretendida indiferencia ("A slow nature such as Maurice's appears insensitive, for it needs time to feel"), el dolor ("He supposed the climax of agony had come"), la expresión física del dolor ("As he laid his head on the pillows a flood of tears oozed from it", pp. 57-58). Forster termina esta fase de despertar con el capítulo 11, y en concreto con una breve reflexión de profunda significación: "After this crisis Maurice became a man." Mediante ella, el lector es consciente de la conexión que ha de existir entre el despertar del individuo a sus sentimientos y la plena conversión a la edad adulta, lo que supondrá la aceptación final de su homosexualidad: "In the afternoon he had a collapse. He remembered that Clive and he had only been together one day! And they spent it careering about like fools instead of in one another's arms!" (p. 78). Esta ausencia de contacto físico entre Maurice y Clive ha llevado a algunos críticos a considerar que se trata de una falta de valor por parte de Forster. Así Norman Page señala:

In a novel intended to strike a blow for the legitimacy of homosexual relationships, this renunciation of the flesh seems curiously self-denying and perhaps represents a failure of nerve on Forster's part (Page 1987: 120).

Sin embargo, Forster mantiene la relación entre ambos en el ámbito espiritual para dar expresión a la doble vía que se abre ante ambos. Por un lado la opción de Clive Durham, expuesto a la preocupación por su posición social y la forma en la que ésta influye en el conflicto moral, afectaría dicha posición. De otro lado, la opción de Maurice, cuya aceptación de su homosexualidad le lleva a identificar a Clive Durham con el ser que había poblado sus sueños infantiles, el amigo que sacrificaría su vida por la suya. No obstante, la aparente dependencia mutua creada por ambos adolece de una 
falta de equilibrio, y es Maurice quien se resentirá en los próximos capítulos, ya que su evolución dependerá del comportamiento de Clive, cuya metamorfosis en heterosexual responde a una combinación de raíces sociales y religiosas, similar a la que lleva a Charles Ryder a abandonar a Sebastian Flyte en favor de su hermana Julia.

El primer reencuentro entre Maurice y Clive se ha de producir en la casa de campo de la familia Durham, simbólico punto de encuentro de tradición y distinción social en declive. Se trata del capítulo 16, que ofrece la escena más pasional entre los dos jóvenes de toda la novela. En efecto, nos sitúa el narrador en la casa, tras una breve introducción sobre el lugar y la partida de Maurice. Estos, tras una escena familiar, se dirigen al estudio e inician una conversación que recupera sus primeros encuentros, sus sentimientos, y establece las bases de su relación. La característica más relevante de esta conversación es que Clive la conduce mientras Maurice responde lacónica y vehementemente. El párrafo más largo de toda la conversación pertenece a Clive y da buena medida de su posición en esta escena:

I'm a bit of an outlaw, I grant, but it serves these people right. As long as they talk of the unspeakable vice of the Greeks they can't expect fair play. It served my mother right when I slipped up to kiss you before dinner. She would have no mercy if she knew, she wouldn't attempt (...) to understand that I feel to you as Pippa [su hermana] to her fiancé, only far more nobly, far more deeply, body and soul, no starved medievalism of course, only a - a particular harmony of body and soul that I don't think women have even guessed (p. 85).

Este idealismo platónico afecta a Maurice a través de Clive, quien desde un principio ha influido en su evolución psicológica. Será en este mismo capítulo cuando, por vez primera, hallemos una voz de disensión en Maurice con respecto al idealismo de Clive: "Why did you mislead me with that rotten Plato? I was still in a muddle" (p. 85). La iniciación sexual de Maurice vendría dada por las palabras de Platón que influyeron en Clive, y que expresan su visión no tan sólo de sus propios sentimientos o emociones, sino que los hace extensivos, como vimos, a los de Maurice. Estas son las palabras de Platón:

El recién iniciado, el que ha contemplado mucho aquellas realidades, cuando ve un rostro divino, que imita bien la belleza verdadera, o un cuerpo igualmente hermoso, primero siente un estremecimiento y le invaden parte de sus temores de entonces; después, dirigiendo sus miradas hacia él, lo venera como a una divinidad (Platón 1987: 87).

Así pues, la afirmación de Clive resulta más racional que pasional. La atracción de la belleza es matizada, a renglón seguido, por la admiración por el hombre, sin explicitar el aspecto al que esta admiración se refiere. Por el contrario, la intervención de Maurice será más pasional al expresar sus sentimientos: “Clive, you're a silly little 
fool, and since you've brought it up I think you're beautiful, the only beautiful person I've ever seen. I love your voice and everything to do with you, down to your clothes or the room you are sitting in. I adore you" (p. 85).

Maurice no se limita a la veneración de la belleza como ideal, sino que ahonda en el lado físco de la relación, e incluso en aspectos de cierta entidad social que sobrepasan los límites del idealismo que propone Clive. La reacción del primero ante estas palabras refleja la complejidad de sus propios sentimientos y, a la discusión sobre la atracción inicial entre ambos, sigue una discusión sobre el arte y la belleza. "Let's change the subject", señala en la página siguiente, asustado por el cariz que la conversación está tomando e incapaz de reaccionar con sinceridad ante las palabras de su Maurice. La escena de amor, con la que concluye el capítulo 16, parece tener un final feliz. El narrador reproduce el ánimo de los dos personajes al partir: "Something of exquisite beauty arose in the mind of each at last", ánimo que comunica al lector un tono de éxtasis sentido por ambos. Sin embargo, y sobre la ilusión del momento, por encima de la visión cegada por la pasión o el idealismo, la distancia entre ellos comienza a hacerse abismal. La concepción que Clive tiene de la apreciación de la belleza y del arte, tal y como refleja en su largo monólogo, sigue fielmente los preceptos de Platón: la diferenciación entre la entrega a las pasiones y la mitificación de la belleza en cualquiera de sus formas, lo que distingue a la mayoría de los seres humanos de la minoría selecta. Por lo tanto, la concepción elitista de Clive responde tanto a su pretendida y pretenciosa actitud de rebelión frente a la sociedad (realiza numerosas referencias a la reacción de su familia o de la sociedad si conociesen la relación existente entre ellos), como en su concepción idealista y elitista del amor hacia la belleza, basada en la prioridad de apreciación estética sobre realización personal.

De otro lado, el segundo paso en la aceptación de su homosexualidad por parte de Maurice tendrá lugar a partir de la consumación física con el guardabosques Scudder. La escena que había liberado sus pasiones y dado lugar a una idealización de una relación puramente física con un ser perteneciente a una clase inferior, se vuelve contra él en el contexto de la Inglaterra de principios de siglo. Maurice se ve inundado por las sospechas sobre el cariz que tomará su inesperada relación con Scudder. Estas se vuelven realidad con la llegada de la escueta nota del criado pidiéndole una cita en Penge. En estos momentos de angustia, Maurice siente todo el peso de la culpabilidad sobre su conciencia, siente haber traicionado a sus amigos en Penge y a su propia familia, que confiaba en él. Es necesario volver a destacar que la culpabilidad de Maurice es estrictamente social. No se ve afectada por remordimientos religiosos ni personales. Maurice se siente culpable por haber traicionado a sus amigos y familia: ha traicionado unas pautas de comportamiento ancestrales, heredadas y aceptadas por ellos. En este momento Maurice acepta la realidad de la sociedad y se siente culpable con respecto a sus normas. De ahí que el sentimiento de culpabilidad se traduzca en un temor al chantaje: "A nice situation! It contained every promise of blackmail (...) He had gone outside his class, and it served him right" (p. 181). 
Sin embargo, la aceptación final de Alec como individuo, no como miembro de una clase inferior, le llevará a su liberación definitiva. Maurice ha llegado al momento crucial en el que ha de aceptar o engañarse a sí mismo para siempre. Un encuentro fortuito con Mr Ducie, el profesor que en la escuela primaria le había explicado los secretos del sexo, le conduce a la liberación final. Mr Ducie reconoce a Maurice, pero no recuerda su nombre. Le confunde con alguno de la larga lista de alumnos que han pasado por la escuela, y que, paulatinamente, se han ido incorporando a la sociedad. La catarsis final se produce con la respuesta de Maurice: “'My name's Scudder.' The correction flew out as the first that occurred to him. It lay ripe to be used, and as he uttered it he knew why” (p. 75), a través de la cual rechaza las enseñanzas de Mr Ducie, sus esfuerzos por convertirlo en uno más, y al tomar el nombre de Scudder acepta, por fin, la realidad de su homosexualidad, su liberación física y la existencia de una human brotherhood, similar a la preconizada por D. H. Lawrence en Aaron's Rod.

Diametralmente opuesta es la consumación de amor homosexual en Radcliffe. Aunque, como habíamos señalado anteriormente, la relación entre Radcliffe y su amante Tolson presenta claros paralelismos con los de Maurice por lo que se refiere a la procedencia social de los protagonistas, el origen de su conflicto queda al margen de lo estrictamente social y se confunde con la personalidad atormentada del protagonista. Stevenson considera que "even the novel's landscape is mildly allegorical, or at least, rather in the manner of Kafka, informed by psychic and emotional qualities" (Stevenson 1986: 132). El personaje de Storey, en el que Stevenson ve claras concomitancias con El idiota de Dovstoievski, es, en realidad, el resultado de la confusión de la posguerra, de los cambios industriales, sociales, religiosos y morales que afectan a la otrora todopoderosa Inglaterra. Por ello, su relación con Tolson es un intento de adaptación social y personal que tiene dramáticas consecuencias, como lo demuestran sus palabras:

'You understand nothing of me!'

'You're afraid!' Leonard's eyes seemed driven back into his skull.

'Afraid!'

'You're afraid of any absolute thing. You're afraid of anything that's complete. That's whole. Anything that takes the whole of you!' (p. 208).

Estas palabras presagian el asesinato de Tolson, al comprender la imposibilidad de alcanzar una unión completa con su amante, la unión entre el espíritu del pasado (el concepto que aparece en Brideshead Revisited definido como "charm") y el cuerpo como símbolo del presente y el futuro. El narrador nos ofrece indicios de un espíritu atormentado en la violencia de los encuentros físicos con Tolson, en los que ésta parte del más débil físicamente: "Leonard swung round and folded his arms round Tolson's neck. He kissed him wildly, pulling Tolson's face ferociously against his mouth" (p. 240). La muerte de Tolson, cuando llega, lo hace con una violencia inusitada como punto álgido de una relación tormentosa: "Leonard lifted the hammer again (...) The 
bone crumbled and a fountain of blood spurted through the dark knots of Tolson's hair..." (p. 311).

\section{Conclusiones}

En el presente artículo hemos analizado la problemática homosexual tal y como se refleja en las novelas de cuatro autores británicos, las relaciones entre éstas, así como su conexión con la realidad social, religiosa, educativa y legal de su época. Nos hemos centrado en aquellos textos que sugieren sutilmente la importancia de la relaciones masculinas, bien abiertamente homosexuales o bien que abogan por la camaradería entre hombres como componente fundamental del desarrollo de la individualidad del protagonista. En el caso de Lawrence, la aséptica descripción de dicha camaradería parece negar la posibilidad de relaciones físicas, aunque el autor favorece de forma innegable la descripción de los protagonistas masculinos frente a los secundarios femeninos, tanto en las relaciones madre-hijo (Sons and Lovers), en la relación no sexual hombre-hombre (Aaron's Rod) o en las relaciones sexuales hombre-mujer (Lady Chatterley's Lover).

El hombre indefenso ante una sociedad intolerante y que necesita regenerarse a través de un amor homosexual platónico es la característica de Clive Durham en Maurice de E. M. Forster. Este homenaje racional y desapasionado a los filósofos griegos, y a la belleza en general, aparece reflejado en Brideshead Revisited de E. Waugh, donde su protagonista se enamora del aristócrata Sebastian Flyte, a pesar de que finalmente se case con su hermana, consciente de la imposibilidad de alcanzar una vida respetable con el primero y deseoso de triunfar en la escena artística británica. Es decir, sucumbe a lo que en la novela se denomina "charm" y renuncia a la pasión, al igual que había hecho Clive Durham, por poder ocupar un puesto relevante en la sociedad. Muy diferente será la decisión de Maurice, al iniciar una relación física no sólo con un hombre, transgrediendo la moral puritana imperante, sino también con un hombre perteneciente a una clase social inferior, con lo que pondrá en peligro su propia situación en la sociedad.

Esta consumación del amor físico llega a su extremo más violento con la novela de David Storey, Radcliffe. En ella la inestabilidad del protagonista se caracteriza por su tendencia a la autodestrucción y a la destrucción de todos los personajes que le rodean, su amante Tolson y su propia familia. Con Radcliffe llegamos al inicio de una nueva era para la temática homosexual en la literatura inglesa. En las décadas anteriores los narradores la habían utilizado en mayor o menor grado dentro de su narrativa, pero nunca abiertamente (la novela de Forster no se publicaría hasta 1971). La liberalización de las costumbres y la eliminación de las barreras lingüísticas sobre el uso de palabras consideradas tabú hasta la abolición del Obscenity Act supuso un cambio drástico que permitiría la expresión literaria (y cinematográfica) de temática clara- 
mente homosexual. Hasta ese momento, las claras coincidencias entre los narradores ingleses eran simplemente la expresión de una imposibilidad de abordar un tema prohibido por la ley y censurado por la sociedad de la época.

\section{Bibliografía}

BRADBURY, M. (ed.) (1990)The Novel Today. Contemporary Writers on Modern Fiction. Londres: Fontana Press.

BEER, J. B. (1962) The Achievements of E. M. Forster. Londres: Chatto \& Windus.

BELL, Q. (1974) Bloomsbury. Londres: Futura Publications.

BURRA, P. (1934) “The Novels of E. M. Forster", Nineteeth Century and After, CXVI.

CREWS, F. (1962) E. M. Forster: The Perils of Humanism. Oxford: Oxford UP.

FORSTER, E. M. (1962) Aspects of the Novel. Harmondsworth: Penguin.

FORSTER, E. M. (1972) Maurice. Harmondsworth: Penguin.

FOUCAULT, M. (1977) Language, Counter-memory, Practice. Nueva York: Ithaca.

FOUCAULT, M. (1978) Historia de la sexualidad I. Madrid: Editorial Siglo XXI.

FOUCAULT, M. (1986) Historia de la sexualidad II. Madrid: Editorial Siglo XXI.

FOUCAULT, M. (1987) Historia de la sexualidad III. Madrid: Editorial Siglo XXI.

FREUD, S. (1988) La interpretación de los sueños. Madrid: Editorial Tecnos.

FURBANK, P. N. (1979) E. M. Forster: A Life. Oxford: Oxford UP.

GOLDENSON, R. \& K. ANDERSON (1994) Dictionary of Sex. Ware: Wordsworth Editions.

GREEN, M. (1984) The English Novel in the Twentieth Century. Londres: Routledge \& Kegan Paul.

HUGUES, G. (1991) Swearing: A Social History of Foul Language, Oaths and Profanity in English. Oxford: Blackwell.

HUXLEY, A. (1955) The Genius and the Goddess. Londres: Chatto \& Windus.

HIMMELFARB, G. (1994) On Looking into the Abyss. New York: Knopf.

ISHERWOOD, C. (1984) All the Conspirators. Londres: Methuen.

KERMODE, F. (1968) "Lawrence and the Apocalyptic Types", Critical Quaterly, X, n. 1-2.

KNIGHT, D. (1990) "Roland Barthes: an intertextual figure", Intertextuality: Theories and Practices, eds. M. Worton \& J. Still. Manchester: Manchester UP.

KRISTEVA, J. (1967) "Bakhtin, le mot, le dialogue et le roman”, Critique 239, pp. 438-65.

KRISTEVA, J. et al. (1968) Théorie d'ensemble. Paris: Éditions du Seuil.

LAWRENCE, D. H. (1948) Sons and Lovers. Harmondsworth: Penguin.

LAWRENCE, D. H. (1950a) Selected Essays. Harmondsworth: Penguin.

LAWRENCE, D. H. (1950b) Aaron's Rod . Harmondsworth: Penguin.

LAWRENCE, D. H. (1960) Lady Chatterley's Lover. Harmondsworth: Penguin. 
LEAVIS, F. R. (1974) D. H. Lawrence, novelista. Barcelona: Barral Editores.

LODGE, D. (1990) After Bakhtin: Essays on Fiction and Society. Londres: Routledge.

PAGE, N. (1987) E. M. Forster. Londres: MacMillan.

PLATON, (1987) Fedro o de la belleza. Madrid: Ediciones Aguilar.

STEVENSON, R. (1986) The British Novel Since the Thirties. Londres: B. T. Batsford.

STONE, W. (1966) The Cave and the Mountain. California: Stanford UP.

STOREY, D. (1965) Radcliffe. Harmondsworth: Penguin.

TRILLING, L. (1970) The Liberal Imagination: Essays on Literature and Society. Harmondsworth: Penguin.

VALDEÓN GARCÍA, R. (1995) Las novelas de E. M. Forster: una revisión intertextual. Oviedo: Servicio de Publicaciones de la Universidad de Oviedo.

VARGAS LLOSA, M. (1994) "Posmodernismo y frivolidad”, El País, 27 de noviembre. WAUGH, E. (1952). Brideshead Revisited. Harmondsworth: Penguin.

WILDE, A. (1964) A Study of E. M. Forster: New York: Nueva York UP.

WORTON, M. \& J. Still. (1990) Intertextuality: theories and practices. Manchester: Manchester UP. 